\title{
Unruh-DeWitt detector response across a Rindler firewall is finite
}

\author{
Jorma Louko \\ School of Mathematical Sciences, University of Nottingham, \\ Nottingham NG7 2RD, U.K. \\ E-mail: jorma.louko@nottingham.ac.uk
}

ABSTRACT: We investigate a two-level Unruh-DeWitt detector coupled to a massless scalar field or its proper time derivative in $(1+1)$-dimensional Minkowski spacetime, in a quantum state whose correlation structure across the Rindler horizon mimics the stationary aspects of a firewall that Almheiri et al. have argued to ensue in an evaporating black hole spacetime. Within first-order perturbation theory, we show that the detector's response on falling through the horizon is sudden but finite. The difference from the Minkowski vacuum response is proportional to $\omega^{-2} \ln (|\omega|)$ for the non-derivative detector and to $\ln (|\omega|)$ for the derivative-coupling detector, both in the limit of a large energy gap $\omega$ and in the limit of adiabatic switching. Adding to the quantum state high Rindler temperature excitations behind the horizon increases the detector's response proportionally to the temperature; this situation has been suggested to model the energetic curtain proposal of Braunstein et al. We speculate that the $(1+1)$-dimensional derivative-coupling detector may be a good model for a non-derivative detector that crosses a firewall in $3+1$ dimensions.

Keywords: Models of Quantum Gravity, Black Holes, Field Theories in Lower Dimensions

ARXIV EPRINT: 1407.6299 


\section{Contents}

1 Introduction 1

2 Two-level UDW detector 3

3 Inertial detector in $1+1$ Minkowski 4

4 A Rindler firewall state $\hat{\rho}_{\mathrm{FW}} \quad 5$

4.1 Definition of $\hat{\rho}_{\mathrm{FW}} \quad 6$

4.2 Properties of $\hat{\rho}_{\mathrm{FW}} \quad 7$

$4.3 \hat{\rho}_{\mathrm{FW}}$ as a firewall model 8

5 Response of an inertial detector in $\hat{\rho}_{\mathrm{FW}} \quad 9$

5.1 Generic horizon-crossing 9

$\begin{array}{ll}5.2 \text { Horizon-crossing at the bifurcation point } & 11\end{array}$

6 Rindler energetic curtain $\quad 11$

$\begin{array}{ll}6.1 \text { The state } & 12\end{array}$

$\begin{array}{lll}6.2 \text { Detector } & 12\end{array}$

$\begin{array}{lll}7 & \text { Summary and concluding remarks } & 13\end{array}$

$\begin{array}{ll}\text { A Asymptotics at large }|\omega| & 15\end{array}$

$\begin{array}{lll}\text { A.1 Minkowski vacuum response } & 15\end{array}$

$\begin{array}{lll}\text { A.2 } & \text { Firewall response } & 16\end{array}$

$\begin{array}{ll}\text { B Evaluation of } \Delta \mathcal{F}^{(0)} \text { and } \Delta \mathcal{F}^{(1)} & 17\end{array}$

$\begin{array}{ll}\text { B.1 } \Delta \mathcal{F}^{(0)} & 18\end{array}$

$\begin{array}{lll}\text { B.2 } \Delta \mathcal{F}^{(1)} & 18\end{array}$

\section{Introduction}

If black hole evaporation is assumed to preserve unitarity, a range of arguments based on quantum correlations [1-3] suggest that physics at the slowly-shrinking horizon may differ significantly from the innocuous picture that underlies Hawking's original derivation of black hole radiation within curved spacetime quantum field theory [4]. In particular, Almheiri et al. [3] have argued that the horizon will be replaced by a region of high curvature, a "firewall", which will destroy any observer who attempts to fall into the black hole. Reviews with extensive references can be found in [5-7].

A key element in the firewall argument as formulated in [3] is that the conventional quantum field theory picture of black hole evaporation involves strong quantum correlations between the black hole interior and exterior, and the assumption of unitary evolution of 
the full system turns out to preclude such correlations. In this paper we examine the consequences of severing closely similar correlations across a Killing horizon in a system in which the requisite quantum state can be readily written down: a conformal scalar field in $1+1$ spacetime dimensions. For concreteness, we take the spacetime to be Minkowski, so that the sense of thermality is that of the Unruh effect of uniform acceleration $[8,9]$, and we induce a firewall by breaking the correlations across the Rindler horizon. The Killing horizon in $(1+1)$-dimensional black hole spacetimes with a Kruskal-like global structure could be treated in the same manner, with similar conclusions.

We shall not attempt to examine how the spacetime geometry might react to the firewall singularity of the scalar field on the Rindler horizon, but we shall examine how the singularity of the scalar field affects a particle detector that falls through the horizon. We consider a two-level Unruh-DeWitt (UDW) detector that couples linearly to the scalar field $[8,10-12]$, and its modification that couples linearly to the proper time derivative of the field [13-18]. The reasons to consider the derivative-coupling detector are twofold. First, for quantum states that are regular in the Hadamard sense [19], the derivative-coupling detector is insensitive to the infrared ambiguity in the Wightman function of the $(1+1)$ dimensional conformal field. Second, the short-distance behaviour of the $(1+1)$-dimensional derivative-coupling UDW detector is similar to that of the $(3+1)$-dimensional UDW detector with a non-derivative coupling [18, 20-22]. We may hence expect a derivative-coupling detector in $1+1$ dimensions to be a good model for a non-derivative detector that crosses a $(3+1)$-dimensional firewall. We recall that the non-derivative UDW detector in $(3+1)$ dimensions models the $\boldsymbol{p} \cdot \boldsymbol{A}$ term by which an atomic electron couples to the quantised electromagnetic field when there is no angular momentum exchange [23, 24].

We shall show that crossing the Rindler firewall has a nonzero and sudden but finite effect on the detector's transition probability, within first-order perturbation theory. In terms of the detector's energy gap $\omega$, the difference from the Minkowski vacuum transition probability is proportional to $\omega^{-2} \ln (|\omega|)$ for the non-derivative detector and to $\ln (|\omega|)$ for the derivative-coupling detector both in the limit of a large energy gap and in the limit of adiabatic switching.

We consider also a generalisation to a quantum state in which Rindler excitations are added behind the Rindler horizon in a way that has been suggested [25] to model the "energetic curtain" of [1] in a black hole spacetime. We show that in this state the response across the horizon is again finite but can be made arbitrarily large by increasing the temperature parameter that characterises the added excitations.

We begin by reviewing in sections 2 and 3 the two-level UDW detector and its derivative-coupling generalisation, coupled to a massless scalar field in $(1+1)$-dimensional Minkowski spacetime. The Rindler firewall quantum state is constructed in section 4 , and we discuss the sense in which it models the stationary aspects of the black hole firewall of [3]. The response of an inertial detector that crosses the Rindler horizon in this state is analysed in section 5, deferring technical steps to two appendices. Section 6 addresses the generalisation to a state in which excitations have been added behind the Rindler horizon. Section 7 presents a summary and concluding remarks, including a discussion of detectors with multiple levels. 
We use metric signature $(-+)$ in which a timelike vector has negative norm squared, and we set $c=\hbar=1$. Spacetime points are denoted by Sans Serif letters $(\mathrm{x})$ and complex conjugation is denoted by an overline.

\section{Two-level UDW detector}

We consider a pointlike two-state UDW detector, moving in a relativistic spacetime on a smooth timelike worldline $\times(\tau)$ parametrised by the proper time $\tau$. The detector's orthonormal energy eigenstates are $|0\rangle_{D}$ and $|\omega\rangle_{D}$, with the respective eigenenergies 0 and $\omega$, where $\omega$ is a real-valued parameter. $|0\rangle_{D}$ is the ground state when $\omega>0$ and the excited state when $\omega<0$. We refer to the detector as a two-level detector. The analysis will cover also the special case $\omega=0$ in which the two states are degenerate in their energy. We start with arbitrary spacetime dimension but will shortly specify to $1+1$.

We couple the detector to a real scalar field $\phi$ via the interaction Hamiltonian

$$
H_{\mathrm{int}}^{(p)}=c \chi(\tau) \mu(\tau) \frac{d^{p}}{d \tau^{p}} \phi(\mathrm{x}(\tau))
$$

where $c$ is a coupling constant, $\mu$ is the detector's monopole moment operator, the parameter $p$ is a non-negative integer, and the switching function $\chi$ specifies how the interaction is switched on an off. We assume $\chi$ to be take non-negative real values and to be smooth with compact support. For $p=0$ the detector couples to the value of the field at the detector's location, and for $p>0$ the detector couples to the $p$ th-order proper time derivative of the field at the detector's location. For the reasons discussed in section 1 we shall mainly be interested in the cases $p=0$, which is the usual UDW detector [8, 10-12], and $p=1$ [13-18], but we shall keep the value of $p$ general until it needs to be specified.

Taking the detector to be initially in the state $|0\rangle_{D}$ and the field to be in a (for the moment pure) state $|\psi\rangle$, and working in first-order perturbation theory in $c$, the probability for the detector to have made a transition to the state $|\omega\rangle_{D}$ after the interaction has ceased can be written for all $p$ by a straightforward adaptation of the $p=0$ analysis $[8,10-12]$. The outcome is

$$
P^{(p)}(\omega)=\left.\left.c^{2}\right|_{D}\langle 0|\mu(0)| \omega\rangle_{D}\right|^{2} \mathcal{F}^{(p)}(\omega),
$$

where the response function $\mathcal{F}^{(p)}$ is given by

$$
\mathcal{F}^{(p)}(\omega)=\int_{-\infty}^{\infty} d \tau^{\prime} \int_{-\infty}^{\infty} d \tau^{\prime \prime} e^{-i \omega\left(\tau^{\prime}-\tau^{\prime \prime}\right)} \chi\left(\tau^{\prime}\right) \chi\left(\tau^{\prime \prime}\right) \partial_{\tau^{\prime}}^{p} \partial_{\tau^{\prime \prime}}^{p} \mathcal{W}\left(\tau^{\prime}, \tau^{\prime \prime}\right)
$$

and the correlation function $\mathcal{W}$ is the pull-back of the Wightman function in the state $|\psi\rangle$ to the detector's worldline,

$$
\mathcal{W}\left(\tau^{\prime}, \tau^{\prime \prime}\right):=\left\langle\psi\left|\phi\left(\times\left(\tau^{\prime}\right)\right) \phi\left(\times\left(\tau^{\prime \prime}\right)\right)\right| \psi\right\rangle
$$

The integrals in (2.3) are understood in the distributional sense, and they are well defined whenever $|\psi\rangle$ is Hadamard [26-29], which we shall assume until this needs to be relaxed in sections 5 and 6 . For mixed states (2.4) is replaced by the pull-back of the mixed state 
Wightman function. From now on we drop the factor $\left.\left.c^{2}\right|_{D}\langle 0|\mu(0)| \omega\rangle_{D}\right|^{2}$ and refer to $\mathcal{F}^{(p)}$ as the transition probability, or as the response.

We now specialise to two spacetime dimensions. Using $\mathcal{W}\left(\tau^{\prime}, \tau^{\prime \prime}\right)=\overline{\mathcal{W}\left(\tau^{\prime \prime}, \tau^{\prime}\right)}$, we may write $\mathcal{F}^{(0)}$ as

$$
\mathcal{F}^{(0)}(\omega)=2 \int_{-\infty}^{\infty} d u \int_{0}^{\infty} d s \chi(u) \chi(u-s) \operatorname{Re}\left[e^{-i \omega s} \mathcal{W}(u, u-s)\right]
$$

where $s=0$ does not require distributional treatment since in two dimensions the short distance singularity of the Wightman function is merely logarithmic [19] and hence integrable. A corresponding expression for $\mathcal{F}^{(1)}$ is [18]

$$
\begin{aligned}
\mathcal{F}^{(1)}(\omega)= & -\omega \Theta(-\omega) \int_{-\infty}^{\infty} d u[\chi(u)]^{2}+\frac{1}{\pi} \int_{0}^{\infty} d s \frac{\cos (\omega s)}{s^{2}} \int_{-\infty}^{\infty} d u \chi(u)[\chi(u)-\chi(u-s)] \\
& +2 \int_{-\infty}^{\infty} d u \int_{0}^{\infty} d s \chi(u) \chi(u-s) \operatorname{Re}\left[e^{-i \omega s}\left(\mathcal{A}(u, u-s)+\frac{1}{2 \pi s^{2}}\right)\right],
\end{aligned}
$$

where $\Theta$ is the Heaviside step function and

$$
\mathcal{A}\left(\tau^{\prime}, \tau^{\prime \prime}\right):=\partial_{\tau^{\prime}} \partial_{\tau^{\prime \prime}} \mathcal{W}\left(\tau^{\prime}, \tau^{\prime \prime}\right)
$$

The last term in (2.6) does not require a distributional treatment at $s=0$ because of the subtraction $\left(2 \pi s^{2}\right)^{-1}$. The price for this subtraction is the emergence of the first two terms in (2.6), neither of which depends on the quantum state of the field or on the detector's motion.

For $\mathcal{F}^{(p)}$ with $p>1$, expressions similar to $(2.5)$ and (2.6) can be obtained by the techniques of $[22]$. We shall consider only $\mathcal{F}^{(0)}$ and $\mathcal{F}^{(1)}$.

\section{Inertial detector in $1+1$ Minkowski}

Let $M$ denote two-dimensional Minkowski spacetime, with the metric $d s^{2}=-d t^{2}+d x^{2}$ in standard global Minkowski coordinates $(t, x)$. We may alternatively use the global null coordinates $u:=t-x$ and $v:=t+x$, in which $d s^{2}=-d u d v$.

We consider a massless scalar field. The Wightman function in the usual Minkowski vacuum $\left|0_{M}\right\rangle$ is

$$
\left\langle 0_{M}\left|\phi(\mathrm{x}) \phi\left(\mathrm{x}^{\prime}\right)\right| 0_{M}\right\rangle=-(4 \pi)^{-1} \ln \left[m_{0}(\epsilon+i \Delta u)\right]-(4 \pi)^{-1} \ln \left[m_{0}(\epsilon+i \Delta v)\right],
$$

where $\Delta u=u-u^{\prime}, \Delta v=v-v^{\prime}, m_{0}$ is a positive constant of dimension inverse length, the logarithms have their principal branch, and the distributional sense is that of $\epsilon \rightarrow 0_{+}$. Because the field is massless, the right-moving and left-moving parts decouple: the $\Delta u$ dependent term in (3.1) comes from the right-movers and the $\Delta v$-dependent term comes from the left-movers.

The constant $m_{0}$ can be understood as an infrared frequency cutoff, and its presence renders the Wightman function ambiguous by an additive real-valued constant. From (2.3) it is seen that $\mathcal{F}^{(0)}$ in $\left|0_{M}\right\rangle$ depends on $m_{0}$ via the additive term

$$
-\frac{\ln \left(m_{0}\right)}{\pi} \int_{0}^{\infty} d s \cos (\omega s) \int_{-\infty}^{\infty} d u \chi(u) \chi(u-s),
$$


and the response of the $p=0$ detector is hence infrared ambiguous. The response of each of the $p>0$ detectors is however infrared unambiguous since the additive constant in the Wightman function drops out on taking the derivatives in (2.3).

For an inertial trajectory, we have $\mathcal{W}\left(\tau^{\prime}, \tau^{\prime \prime}\right)=-(2 \pi)^{-1} \ln \left[m_{0}\left(\epsilon+i\left(\tau^{\prime}-\tau^{\prime \prime}\right)\right)\right]$, and $(2.5)$ and (2.6) give

$$
\begin{aligned}
\operatorname{in} \mathcal{F}_{\left|0_{M}\right\rangle}^{(0)}(\omega)= & -\int_{0}^{\infty} d s\left[\frac{1}{2} \sin (\omega s)+\pi^{-1} \cos (\omega s) \ln \left(m_{0} s\right)\right] \int_{-\infty}^{\infty} d u \chi(u) \chi(u-s), \\
\operatorname{in} \mathcal{F}_{\left|0_{M}\right\rangle}^{(1)}(\omega)= & -\omega \Theta(-\omega) \int_{-\infty}^{\infty} d u[\chi(u)]^{2} \\
& +\frac{1}{\pi} \int_{0}^{\infty} d s \frac{\cos (\omega s)}{s^{2}} \int_{-\infty}^{\infty} d u \chi(u)[\chi(u)-\chi(u-s)]
\end{aligned}
$$

where the left superscript in indicates that the trajectory is inertial. At a large energy gap, $|\omega| \rightarrow \infty$, we show in appendix A that

$$
\begin{aligned}
& \text { in } \mathcal{F}_{\left|0_{M}\right\rangle}^{(0)}(\omega)=-\frac{\Theta(-\omega)}{\omega}\left[\int_{-\infty}^{\infty} d u[\chi(u)]^{2}+\frac{1}{\omega^{2}} \int_{-\infty}^{\infty} d u\left[\chi^{\prime}(u)\right]^{2}\right. \\
& \left.+\cdots+\frac{1}{\omega^{2 k}} \int_{-\infty}^{\infty} d u\left[\chi^{(k)}(u)\right]^{2}\right]+O\left(\frac{1}{\omega^{2 k+3}}\right), \\
& { }^{\text {in }} \mathcal{F}_{\left|0_{M}\right\rangle}^{(1)}(\omega)=-\omega \Theta(-\omega) \int_{-\infty}^{\infty} d u[\chi(u)]^{2}+O\left(\frac{1}{\omega^{2 k}}\right) \text {, }
\end{aligned}
$$

for all positive integers $k$. The infrared ambiguity of $\mathcal{F}_{\left|0_{M}\right\rangle}^{(0)}$ does not show up in the large $|\omega|$ form (3.4a) because the ambiguous contribution (3.2) falls off faster than any inverse power of $\omega$.

We are also interested in the adiabatic limit of slow switching and long detection. We implement this by writing $\chi(\tau)=g(\alpha \tau)$ where $\alpha$ is a positive parameter, $g$ is a fixed switching function, and the limit of interest is $\alpha \rightarrow 0_{+}$. Changing integration variables by $u=v / \alpha$ and $s=r / \alpha$, comparing with (3.4), and assuming $\omega \neq 0$, we see that

$$
\begin{aligned}
& { }^{\text {in }} \mathcal{F}_{\left|0_{M}\right\rangle}^{(0)}(\omega)=-\frac{\Theta(-\omega)}{\omega}\left[\alpha^{-1} \int_{-\infty}^{\infty} d v[g(v)]^{2}+\frac{\alpha}{\omega^{2}} \int_{-\infty}^{\infty} d v\left[g^{\prime}(v)\right]^{2}\right. \\
& \left.+\cdots+\frac{\alpha^{2 k-1}}{\omega^{2 k}} \int_{-\infty}^{\infty} d v\left[g^{(k)}(v)\right]^{2}\right]+O\left(\alpha^{2 k+1}\right), \\
& \operatorname{in} \mathcal{F}_{\left|0_{M}\right\rangle}^{(1)}(\omega)=-\omega \Theta(-\omega) \alpha^{-1} \int_{-\infty}^{\infty} d v[g(v)]^{2}+O\left(\alpha^{2 k}\right),
\end{aligned}
$$

for all positive integers $k$. The probability of an excitation hence vanishes in the adiabatic limit, while the probablity of a de-exitation is proportional to $\alpha^{-1}$, that is, proportional to the total detection time.

\section{A Rindler firewall state $\hat{\rho}_{\mathrm{FW}}$}

We continue to consider a massless scalar field $\phi$ on two-dimensional Minkowski spacetime $M$, in the notation of section 3 . In this section we construct a mixed state $\hat{\rho}_{\text {FW }}$ in 


\begin{tabular}{|l|c|c|}
\hline Quadrant & Range in $(t, x)$ & Range in $(u, v)$ \\
\hline F: future & $t>|x|$ & $u>0, v>0$ \\
P: past & $t<-|x|$ & $u<0, v<0$ \\
R: right & $x>|t|$ & $u<0, v>0$ \\
L: left & $x<-|t|$ & $u>0, v<0$ \\
\hline
\end{tabular}

Table 1. The four open quadrants of two-dimensional Minkowski spacetime.

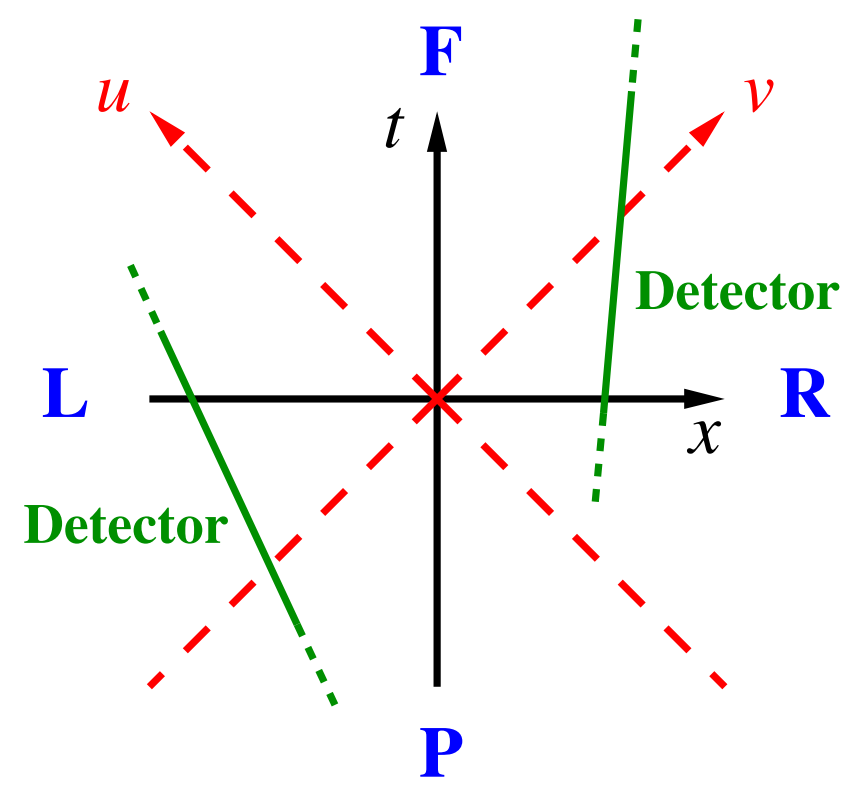

Figure 1. $(1+1)$-dimensional Minkowski spacetime. The dashed (red) lines show the Rindler horizon $t^{2}-x^{2}=0$, which separates the four quadrants $\mathbf{F}, \mathbf{P}, \mathbf{R}$ and $\mathbf{L}$ as summarised in table 1 . Also shown are the worldlines (green) of two inertial detectors, each of which operates for a finite interval of time and crosses during that interval exactly one branch of the Rindler horizon.

which correlations that are present in $\left|0_{M}\right\rangle$ have been severed across the Rindler horizon. We discuss the sense in which $\hat{\rho}_{\mathrm{FW}}$ models the stationary aspects of a similar severing that has been argued in [3] to ensue dynamically in an evaporating black hole spacetime.

\subsection{Definition of $\hat{\rho}_{\mathrm{FW}}$}

Recall that the Rindler horizon in $M$ is at $t^{2}-x^{2}=0$, or in terms of the null coordinates, at $u v=0$. We denote the future, past, right and left open quadrants separated by the Rindler horizon by respectively $\mathbf{F}, \mathbf{P}, \mathbf{R}$ and $\mathbf{L}$, as summarised in table 1 and shown in figure 1.

Recall also that the restriction of $\left|0_{M}\right\rangle$ to $\mathbf{R}$ is a mixed state whose density matrix $\rho_{\mathbf{R}}$ is thermal in temperature $(2 \pi)^{-1}$ with respect to the boost Killing vector $\xi:=x \partial_{t}+t \partial_{x}=$ $-u \partial_{u}+v \partial_{v}$, which is timelike and future-pointing in $\mathbf{R}[8,11,12]$. Similarly, the restriction of $\left|0_{M}\right\rangle$ to $\mathbf{L}$ is a mixed state whose density matrix $\rho_{\mathbf{L}}$ is thermal in temperature $(2 \pi)^{-1}$ with respect to the boost Killing vector $-\xi$, which is timelike and future-pointing in $\mathbf{L}$. 


\begin{tabular}{|c|c|}
\hline Quadrant pairs & $\operatorname{Tr}\left(\phi(\mathrm{x}) \phi\left(\mathrm{x}^{\prime}\right) \tilde{\rho}_{\mathrm{FW}}\right)$ \\
\hline $\begin{array}{l}\mathbf{P} \text { and } \mathbf{R} \\
\mathbf{L} \text { and } \mathbf{F}\end{array}$ & $-(4 \pi)^{-1} \ln \left[m_{0}(\epsilon+i \Delta u)\right]$ \\
\hline $\mathbf{P}$ and $\mathbf{L}$ & $-(4 \pi)^{-1} \ln \left[m_{0}(\epsilon+i \Delta v)\right]$ \\
$\mathbf{R}$ and $\mathbf{F}$ & 0 \\
\hline $\begin{array}{l}\mathbf{R} \text { and } \mathbf{L} \\
\mathbf{P} \text { and } \mathbf{F}\end{array}$ \\
\hline
\end{tabular}

Table 2. The table shows $\operatorname{Tr}\left(\phi(\mathrm{x}) \phi\left(\mathrm{x}^{\prime}\right) \tilde{\rho}_{\mathrm{FW}}\right)$ when $\mathrm{x}$ and $\mathrm{x}^{\prime}$ are in distinct quadrants of $\mathbf{F} \cup \mathbf{P} \cup \mathbf{R} \cup \mathbf{L}$. In the pairs $(\mathbf{P}, \mathbf{R})$ and $(\mathbf{L}, \mathbf{F})$, the two quadrants are causally correlated for right-movers and $\operatorname{Tr}\left(\phi(\mathrm{x}) \phi\left(\mathrm{x}^{\prime}\right) \tilde{\rho}_{\mathrm{FW}}\right)$ contains only the right-mover contribution to $\left\langle 0_{M}\left|\phi(\mathrm{x}) \phi\left(\mathrm{x}^{\prime}\right)\right| 0_{M}\right\rangle$. In the pairs $(\mathbf{P}, \mathbf{L})$ and $(\mathbf{R}, \mathbf{F})$, the two quadrants are causally correlated for left-movers and $\operatorname{Tr}\left(\phi(\mathrm{x}) \phi\left(\mathrm{x}^{\prime}\right) \tilde{\rho}_{\mathrm{FW}}\right)$ contains only the left-mover contribution to $\left\langle 0_{M}\left|\phi(\mathrm{x}) \phi\left(\mathrm{x}^{\prime}\right)\right| 0_{M}\right\rangle$. In the pairs $(\mathbf{R}, \mathbf{L})$ and $(\mathbf{P}, \mathbf{F})$, the two quadrants have no causal correlation and $\operatorname{Tr}\left(\phi(\mathrm{x}) \phi\left(\mathrm{x}^{\prime}\right) \tilde{\rho}_{\mathrm{FW}}\right)$ vanishes. When $\mathrm{x}$ and $\mathrm{x}^{\prime}$ are in the same quadrant, $\operatorname{Tr}\left(\phi(\mathrm{x}) \phi\left(\mathrm{x}^{\prime}\right) \tilde{\rho}_{\mathrm{FW}}\right)=\left\langle 0_{M}\left|\phi(\mathrm{x}) \phi\left(\mathrm{x}^{\prime}\right)\right| 0_{M}\right\rangle$.

Now, consider on $\mathbf{R} \cup \mathbf{L}$ the mixed state whose density matrix is $\rho_{\mathrm{FW}}:=\rho_{\mathbf{R}} \otimes \rho_{\mathbf{L}}$. For any observable whose support is contained in $\mathbf{R}$, the expectation value in $\rho_{\mathrm{FW}}$ is identical to the expectation value in $\left|0_{M}\right\rangle$, and similarly for any observable whose support is contained in $\mathbf{L}$. However, $\rho_{\mathrm{FW}}$ contains no correlations between $\mathbf{R}$ and $\mathbf{L}$ : all the correlations between $\mathbf{R}$ and $\mathbf{L}$ that are present in $\left|0_{M}\right\rangle[8,11,12,30]$ have been severed in $\rho_{\mathrm{FW}}$.

We wish to extend $\rho_{\text {FW }}$ beyond $\mathbf{R} \cup \mathbf{L}$. There exists a unique extension to $\mathbf{F} \cup \mathbf{P} \cup \mathbf{R} \cup \mathbf{L}$ : because the field is massless, the left-moving part of the field propagates into $\mathbf{F}$ only from $\mathbf{R}$ and into $\mathbf{P}$ only from $\mathbf{L}$, while the right-moving part of the field propagates into $\mathbf{F}$ only from $\mathbf{L}$ and into $\mathbf{P}$ only from $\mathbf{R}$. We denote this extension by $\tilde{\rho}_{\mathrm{FW}}$. The Wightman function in $\tilde{\rho}_{\mathrm{FW}}$, given by $\operatorname{Tr}\left(\phi(\mathrm{x}) \phi\left(\mathrm{x}^{\prime}\right) \tilde{\rho}_{\mathrm{FW}}\right)$, is equal to $\left\langle 0_{M}\left|\phi(\mathrm{x}) \phi\left(\mathrm{x}^{\prime}\right)\right| 0_{M}\right\rangle$ when $\mathrm{x}$ and $\mathrm{x}^{\prime}$ are in the same quadrant, but not when $x$ and $x^{\prime}$ are in distinct quadrants, as collected in table 2 .

Extending $\operatorname{Tr}\left(\phi(\mathbf{x}) \phi\left(\mathrm{x}^{\prime}\right) \tilde{\rho}_{\mathrm{FW}}\right)$ from $\mathbf{F} \cup \mathbf{P} \cup \mathbf{R} \cup \mathbf{L}$ to all of Minkowski requires additional input on the Rindler horizon. We adopt the extension that is minimal in the sense that it has no distributional support at the Rindler horizon. This extension is unique: we denote it by $\operatorname{Tr}\left(\phi(\mathrm{x}) \phi\left(\mathrm{x}^{\prime}\right) \hat{\rho}_{\mathrm{FW}}\right)$, and we interpret it as the Wightman function of a mixed state whose density matrix we denote by $\hat{\rho}_{\mathrm{FW}}$.

\subsection{Properties of $\hat{\rho}_{\mathrm{FW}}$}

By construction, $\hat{\rho}_{\mathrm{FW}}$ is indistinguishable from $\left|0_{M}\right\rangle$ for any operator whose support is contained in any one of the four quadrants $\mathbf{F}, \mathbf{P}, \mathbf{R}$, and $\mathbf{L}$. In particular, the restriction of $\hat{\rho}_{\text {FW }}$ to any one of the quadrants is Hadamard and has a vanishing stress-energy tensor.

The restriction of $\hat{\rho}_{\mathrm{FW}}$ to any one of the quadrants is also invariant under the Lorentz boosts generated by the Killing vector $\xi$. The restrictions of $\hat{\rho}_{\mathrm{FW}}$ to $\mathbf{R}$ and $\mathbf{L}$ are hence stationary with respect to Rindler time translations, and observers on the uniformlyaccelerated world lines $x^{2}-t^{2}=a^{-2}$, where the positive constant $a$ is the acceleration, will experience the usual Unruh effect, in temperature $a /(2 \pi)[8,9]$. The restrictions of $\hat{\rho}_{\mathrm{FW}}$ 
to $\mathbf{R}$ and $\mathbf{L}$ are also invariant with respect to Minkowski time translations in a local sense, but not globally, since Minkowski time translations necessarily map $\mathbf{R}$ and $\mathbf{L}$ to regions that intersect the Rindler horizons.

The Wightman function of $\hat{\rho}_{\mathrm{FW}}$ is by construction a well-defined distribution everywhere, including the Rindler horizon. The response of a horizon-crossing detector in the state $\hat{\rho}_{\mathrm{FW}}$ is hence well defined by (2.3). As the Wightman function is not invariant under Lorentz boosts generated by $\xi$ when the two arguments are in distinct quadrants of the pairs $(\mathbf{P}, \mathbf{R}),(\mathbf{P}, \mathbf{L}),(\mathbf{R}, \mathbf{F})$ or $(\mathbf{L}, \mathbf{F})$, we may expect Lorentz-noninvariance in the response of a detector that crosses exactly one branch of the Rindler horizon, and we may expect this noninvariance to be associated with the infrared cutoff $m_{0}$ : this is what will be found in section 5 .

The Wightman function of $\hat{\rho}_{\mathrm{FW}}$ is not Hadamard at the Rindler horizon. We shall not attempt to examine in which sense $\hat{\rho}_{\text {FW }}$ may be definable on the Rindler horizon beyond its Wightman function, and in particlar we shall not attempt to define a stress-energy tensor for $\hat{\rho}_{\mathrm{FW}}$ on the Rindler horizon. We shall return to this point in section 7 .

\section{3 $\hat{\rho}_{\mathrm{FW}}$ as a firewall model}

$\hat{\rho}_{\mathrm{FW}}$ contains by construction no correlations between the spacetime regions $\mathbf{R}$ and $\mathbf{L}$. We may view $\hat{\rho}_{\mathrm{FW}}$ as the minimal modification of $\left|0_{M}\right\rangle$ in which the correlations between $\mathbf{R}$ and $\mathbf{L}[8,11,12,30]$ have been fully severed. The severing has made $\hat{\rho}_{\mathrm{FW}}$ singular on the Rindler horizon, but with a Wightman function that is still a well-defined distribution.

In the spacetime of an evaporating black hole, the conventional quantum field theory picture implies that the field develops strong correlations between the interior and exterior of the hole, closely similar to the correlations in $\left|0_{M}\right\rangle$ across the Rindler horizon $[4,8,11,12]$. It is argued in [3] that these correlations cannot be maintained if the quantum evolution of the full system is assumed unitary. It is further argued in [3] that breaking the correlations will replace the horizon by a firewall, a region of high curvature, which will destroy any observer who attempts to fall into the black hole. Our state $\hat{\rho}_{\mathrm{FW}}$ models within Minkowski spacetime quantum field theory the severed quantum correlations across the firewall of [3]. A detector crossing the Rindler horizon from $\mathbf{R}$ or $\mathbf{L}$ to $\mathbf{F}$, in the state $\hat{\rho}_{\mathrm{FW}}$, models a detector crossing the firewall of [3] as long as the shrinking black hole horizon has not yet become gravitationally singular due to any back-reaction from the stress-energy of the firewall quantum state.

In summary, $\hat{\rho}_{\mathrm{FW}}$ models the stationary aspects of the black hole firewall of [3]. The relevant sense of stationarity in $\hat{\rho}_{\mathrm{FW}}$ is with respect to Lorentz boosts. The Lorentznonivariance of $\hat{\rho}_{\mathrm{FW}}$ means that the modelling will not be fully stationary, but this nonstationarity is associated with the infrared cutoff $m_{0}$ and we will see that it will not be significant for the conclusions.

We emphasise that we shall not attempt to model how the severing of the quantum correlations in the firewall state of [3] may arise through the evolution of the full quantum system, nor shall we attempt to model how the spacetime reacts to the singularity in the firewall state. Also, we shall not attempt to discuss in detail the near-horizon phenomena 
proposed in $[1,2]$, but we shall consider in section 6 a generalisation of $\hat{\rho}_{\mathrm{FW}}$ that has been suggested [25] to model the energetic curtain of [1].

We refer to $\hat{\rho}_{\mathrm{FW}}$ as a Rindler firewall state.

\section{$5 \quad$ Response of an inertial detector in $\hat{\rho}_{\mathrm{FW}}$}

In this section we examine the response of the two-level detector of section 2 when it crosses the Rindler horizon and the field is in the Rindler firewall state $\hat{\rho}_{\mathrm{FW}}$. We take the detector to be inertial and to cross the horizon exactly once during the time that it operates. Subsection 5.1 considers the generic case, shown in figure 1, in which the horizoncrossing occurs away from the bifurcation point $(t, x)=(0,0)$. Crossing from $\mathbf{R}$ or $\mathbf{L}$ to $\mathbf{F}$ models crossing the black hole firewall of [3], but we shall see that crossing from $\mathbf{P}$ to $\mathbf{R}$ or $\mathbf{L}$ yields an identical response. The special case of a detector that goes through the bifurcation point is treated in subsection 5.2.

\subsection{Generic horizon-crossing}

In this subsection we consider an inertial detector that crosses exactly one branch of the horizon during the time that it operates, as shown in figure 1. We introduce the parameter $\eta$ that takes the value 1 if this this branch is the left-going branch, $v=0$, and the value -1 if this branch is the right-going branch, $u=0$. We write the detector's velocity vector as $\cosh (\beta) \partial_{t}+\sinh (\beta) \partial_{x}$, where $\beta \in \mathbb{R}$ is the rapidity with respect to the Lorentz-frame $(t, x)$, and we parametrise the trajectory so that the horizon-crossing occurs at $\tau=0$.

Let in $\mathcal{F}_{\left|0_{M}\right\rangle}^{(p)}$ and in $\mathcal{F}_{\mathrm{FW}}^{(p)}$ denote the response of the inertial detector in the respective states $\left|0_{M}\right\rangle$ and $\hat{\rho}_{\text {FW. }}$. Using (2.3), and the Wightman functions given in (3.1) and in table 2, we see that the difference $\Delta \mathcal{F}^{(p)}:={ }^{\text {in }} \mathcal{F}_{\mathrm{FW}}^{(p)}-{ }^{\text {in }} \mathcal{F}_{\left|0_{M}\right\rangle}^{(p)}$ is given by

$$
\Delta \mathcal{F}^{(p)}(\omega)=\int_{-\infty}^{\infty} d \tau^{\prime} \int_{-\infty}^{\infty} d \tau^{\prime \prime} e^{-i \omega\left(\tau^{\prime}-\tau^{\prime \prime}\right)} \chi\left(\tau^{\prime}\right) \chi\left(\tau^{\prime \prime}\right) \partial_{\tau^{\prime}}^{p} \partial_{\tau^{\prime \prime}}^{p} \Delta \mathcal{W}\left(\tau^{\prime}, \tau^{\prime \prime}\right)
$$

where

$$
\Delta \mathcal{W}\left(\tau^{\prime}, \tau^{\prime \prime}\right)= \begin{cases}(4 \pi)^{-1} \ln \left[m_{0} e^{\eta \beta}\left(\tau^{\prime}-\tau^{\prime \prime}\right)\right]+\frac{1}{8} i & \text { for } \tau^{\prime}>0>\tau^{\prime \prime} \\ (4 \pi)^{-1} \ln \left[m_{0} e^{\eta \beta}\left(\tau^{\prime \prime}-\tau^{\prime}\right)\right]-\frac{1}{8} i & \text { for } \tau^{\prime \prime}>0>\tau^{\prime} \\ 0 & \text { otherwise }\end{cases}
$$

$\Delta \mathcal{F}^{(p)}$ is well defined and finite for each $p$ : the derivatives in (5.1) are distributional but the integrals exist and are finite since $\chi$ is by assumption smooth and of compact support. 
We now specialise to $p=0$ and $p=1$. It is shown in appendix B that

$$
\begin{aligned}
\Delta \mathcal{F}^{(0)}(\omega)= & \int_{0}^{\infty} d s\left[\frac{1}{4} \sin (\omega s)+(2 \pi)^{-1} \cos (\omega s) \ln \left(m_{0} e^{\eta \beta} s\right)\right] \int_{0}^{s} d u \chi(u) \chi(u-s), \\
\Delta \mathcal{F}^{(1)}(\omega)= & \frac{[\chi(0)]^{2}}{2 \pi} \ln \left(|\omega| e^{\gamma-1} e^{-\eta \beta} / m_{0}\right) \\
& +\frac{1}{2 \pi} \int_{0}^{\infty} d s \cos (\omega s)\left\{\frac{\chi(0)[\chi(0)-\chi(s)-\chi(-s)]}{s}\right. \\
& \left.+\frac{1}{s^{2}} \int_{0}^{s} d u \chi(u) \chi(u-s)\right\} \quad \text { for } \omega \neq 0, \\
\Delta \mathcal{F}^{(1)}(0)= & \frac{\chi(0)}{4 \pi} \int_{0}^{\infty} d s \ln \left(e m_{0} e^{\eta \beta} s\right)\left[\chi^{\prime}(s)-\chi^{\prime}(-s)\right] \\
& +\frac{1}{2 \pi} \int_{0}^{\infty} d s\left\{-\frac{\chi(0)[\chi(s)+\chi(-s)]}{2 s}+\frac{1}{s^{2}} \int_{0}^{s} d u \chi(u) \chi(u-s)\right\},
\end{aligned}
$$

where $\gamma$ is Euler's constant.

Several observations are in order. For properties that hold for both of $\Delta \mathcal{F}^{(0)}$ and $\Delta \mathcal{F}^{(1)}$, we refer to the two by $\Delta \mathcal{F}$.

First, $\Delta \mathcal{F}$ is even in $\omega$ : the firewall has identical effects on probabilities of excitation and de-excitation.

Second, $\Delta \mathcal{F}$ is invariant under $\chi(\tau) \rightarrow \chi(-\tau)$ : the firewall effect is invariant under a future-past reflection about the horizon-crossing moment.

Third, $\Delta \mathcal{F}$ depends on the infrared cutoff $m_{0}$. It also depends on the trajectory's rapidity parameter $\beta$ and is hence not Lorentz invariant. We shall shortly see that these effects are subdominant in the limit of a large energy gap and in the limit of adiabatic switching, but we may note here that the Lorentz noninvariance is directly connected to the cutoff: the term that depends on $m_{0}$ and $\beta$ is

$$
\begin{array}{ll}
p=0: & \frac{\ln \left(m_{0} e^{\eta \beta}\right)}{2 \pi} \int_{0}^{\infty} d s \cos (\omega s) \int_{0}^{s} d u \chi(u) \chi(u-s), \\
p=1: & -(2 \pi)^{-1}[\chi(0)]^{2} \ln \left(m_{0} e^{\eta \beta}\right),
\end{array}
$$

which shows that increasing (respectively decreasing) the detector's velocity towards the horizon has the effect of increasing (decreasing) the effective infrared cutoff $m_{0} e^{\eta \beta}$ by precisely the appropriate Doppler shift factor. Note also that for $p=0$ the ambiguous term (5.4a) comes from a finite neighbourhood of the horizon-crossing moment, while for $p=1$ the ambiguous term (5.4b) comes strictly from the horizon-crossing moment and vanishes iff $\chi(0)=0$.

Fourth, $\Delta \mathcal{F}$ is nonvanishing whenever $\chi$ has support both before and after the horizoncrossing, regardless whether the detector is in operation at the horizon-crossing moment. 
Fifth, we show in appendix A that $\Delta \mathcal{F}$ has the large $|\omega|$ form

$$
\begin{aligned}
\Delta \mathcal{F}^{(0)}(\omega)= & \frac{1}{2 \pi} \ln \left(|\omega| e^{\gamma-1} e^{-\eta \beta} / m_{0}\right)\left(\frac{[\chi(0)]^{2}}{\omega^{2}}+\frac{\left[\chi^{\prime}(0)\right]^{2}-2 \chi(0) \chi^{\prime \prime}(0)}{\omega^{4}}+O\left(\omega^{-6}\right)\right) \\
& +\frac{2 \chi(0) \chi^{\prime \prime}(0)-\left[\chi^{\prime}(0)\right]^{2}}{6 \pi \omega^{4}}+O\left(\omega^{-6}\right) \\
\Delta \mathcal{F}^{(1)}(\omega)= & \frac{[\chi(0)]^{2}}{2 \pi} \ln \left(|\omega| e^{\gamma-1} e^{-\eta \beta} / m_{0}\right)+\frac{4 \chi(0) \chi^{\prime \prime}(0)+\left[\chi^{\prime}(0)\right]^{2}}{12 \pi \omega^{2}}+O\left(\omega^{-4}\right) .
\end{aligned}
$$

In the special case $\chi(0)=0$, all the terms shown in (5.5) vanish and the first potentially nonvanishing terms are

$$
\begin{aligned}
& \Delta \mathcal{F}^{(0)}(\omega)=\frac{1}{2 \pi} \ln \left(|\omega| e^{\gamma-1} e^{-\eta \beta} / m_{0}\right)\left(\frac{\left[\chi^{\prime \prime}(0)\right]^{2}}{\omega^{6}}+O\left(\omega^{-8}\right)\right)-\frac{8\left[\chi^{\prime \prime}(0)\right]^{2}}{30 \pi \omega^{6}}+O\left(\omega^{-8}\right), \\
& \Delta \mathcal{F}^{(1)}(\omega)=\frac{\left[\chi^{\prime \prime}(0)\right]^{2}}{40 \pi \omega^{4}}+O\left(\omega^{-6}\right) .
\end{aligned}
$$

The dominant effect at large $|\omega|$ comes hence from the horizon-crossing moment. If $\chi$ and all its derivatives vanish at the horizon-crossing, $\Delta \mathcal{F}$ vanishes at $|\omega| \rightarrow \infty$ faster than any inverse power of $\omega$.

Sixth, to analyse the adiabatic limit, we write $\chi(\tau)=g(\alpha \tau)$ where $\alpha$ is a positive parameter, $g$ is a fixed switching function, and we are interested in the limit $\alpha \rightarrow 0_{+}$. Changing in (5.3) integration variables by $u=v / \alpha$ and $s=r / \alpha$, and assuming $\omega \neq 0$, we see that the asymptotic formulas are obtained from (5.5) and (5.6) by multiplying $\Delta \mathcal{F}^{(0)}$ by $\alpha^{-2}$ and making the replacements $\chi \rightarrow g, \omega \rightarrow \omega / \alpha$ and $m_{0} \rightarrow m_{0} / \alpha$. The dominant effect in $\Delta \mathcal{F}$ in the adiabatic limit hence comes from the horizon-crossing moment, and if the detector operates at this moment, the leading term in $\Delta \mathcal{F}$ is independent of $\alpha$ and equal to the leading term shown in (5.5). When the Minkowski vacuum contribution (3.5) to the response is included, we see that if the detector operates at the horizon-crossing moment, the firewall gives the leading adiabatic contribution to the excitation probability and the next-to-leading adiabatic contribution to the de-excitation probability.

\subsection{Horizon-crossing at the bifurcation point}

In the special case in which the detector crosses the horizon at the bifurcation point, $\Delta \mathcal{F}$ is given by summing over the two values of $\eta$ in (5.3). $\Delta \mathcal{F}$ is hence obtained from (5.3) by setting $\eta=0$ and including an overall multiplicative factor 2 . The only qualitatively new property is that $\Delta \mathcal{F}$ is now independent of $\beta$ and hence Lorentz invariant.

\section{$6 \quad$ Rindler energetic curtain}

In this section we consider a generalisation of $\hat{\rho}_{\mathrm{FW}}$ whose restriction to $\mathbf{L}$ is thermal with respect to the future-pointing Killing vector $-\xi$ in the (dimensionless) temperature $T>0$, and an inertial detector crossing the Rindler horizon from $\mathbf{R}$ to $\mathbf{F}$. It has been suggested [25] that at $T \gg(2 \pi)^{-1}$ this system models a detector crossing the energetic curtain of [1] in a black hole spacetime. 


\subsection{The state}

Let $\widehat{M}$ denote an auxiliary $(1+1)$-dimensional Minkowski spacetime, with the metric $\widehat{d s^{2}}=-d \hat{u} d \hat{v}$ in the dimensionless null coordinates $(\hat{u}, \hat{v})$. For a massless scalar field on $\widehat{M}$, the Wightman function in a thermal state of temperature $T>0$ with respect to the normalised time translation Killing vector $\hat{\xi}:=\partial_{\hat{u}}+\partial_{\hat{v}}$ reads [18]

$$
\hat{G}_{T}\left(\left(\hat{u}^{\prime}, \hat{v}^{\prime}\right),\left(\hat{u}^{\prime \prime}, \hat{v}^{\prime \prime}\right)\right)=-(4 \pi)^{-1} \ln \{-\sinh [\pi T(\Delta \hat{u}-i \epsilon)] \sinh [\pi T(\Delta \hat{v}-i \epsilon)]\},
$$

where $\Delta \hat{u}=\hat{u}^{\prime}-\hat{u}^{\prime \prime}, \Delta \hat{v}=\hat{v}^{\prime}-\hat{v}^{\prime \prime}$, the logarithm has its principal branch and the distributional sense is that of $\epsilon \rightarrow 0_{+}$. Note that the temperature parameter $T$ is dimensionless since $\hat{u}$ and $\hat{v}$ are dimensionless.

We map $\widehat{M}$ conformally to the region $\mathbf{L}$ in the $(1+1)$-dimensional Minkowski spacetime $M$ of section 3 , by $u=m_{0}^{-1} e^{\hat{u}}$ and $v=-m_{0}^{-1} e^{-\hat{v}}$, so that $d s^{2}=-d u d v=(-u v) \widehat{d s^{2}}$. The push-forward of $\hat{\xi}$ to $\mathbf{L}$ is the future-pointing boost Killing vector $u \partial_{u}-v \partial_{v}=-\xi$, and the push-forward of $\hat{G}_{T}$ is $G_{T}^{\mathrm{L}}\left(\mathrm{x}, \mathrm{x}^{\prime}\right)+\frac{1}{4} T \ln \left(m_{0}^{4} u^{\prime} u^{\prime \prime} v^{\prime} v^{\prime \prime}\right)$, where

$$
\begin{aligned}
G_{T}^{\mathbf{L}}\left(\mathrm{x}, \mathrm{x}^{\prime}\right)= & -(4 \pi)^{-1} \ln \left\{\epsilon+i\left[\left(m_{0} u^{\prime}\right)^{2 \pi T}-\left(m_{0} u^{\prime \prime}\right)^{2 \pi T}\right]\right\} \\
& -(4 \pi)^{-1} \ln \left\{\epsilon+i\left[\left(-m_{0} v^{\prime \prime}\right)^{2 \pi T}-\left(-m_{0} v^{\prime}\right)^{2 \pi T}\right]\right\} .
\end{aligned}
$$

As the term $\frac{1}{4} T \ln \left(m_{0}^{4} u^{\prime} u^{\prime \prime} v^{\prime} v^{\prime \prime}\right)$ is regular in $\mathbf{L}$ and satisfies the field equation there, we may drop this term and define in $\mathbf{L}$ a quantum state whose Wightman function equals $G_{T}^{\mathbf{L}}$. We denote the density matrix of this state by $\rho_{\mathbf{L}, T}$. Note that $\rho_{\mathbf{L},(2 \pi)^{-1}}=\rho_{\mathbf{L}}$.

Now, the right-mover part of $G_{T}^{\mathbf{L}}$ continues without singularities from $\mathbf{L}$ to $\mathbf{F}$, and the left-mover part continues without singularities from $\mathbf{L}$ to $\mathbf{P}$. We may hence define on $M$ a state by starting from $\rho_{\mathbf{R}} \otimes \rho_{\mathbf{L}, \mathrm{T}}$ on $\mathbf{R} \cup \mathbf{L}$ and extending to all of $M$ by causal propagation as in section 4 . We denote the density matrix of this state by $\hat{\rho}_{\mathrm{EC}, T}$. By construction, $\hat{\rho}_{\mathrm{EC},(2 \pi)^{-1}}=\hat{\rho}_{\mathrm{FW}}$. We regard $\hat{\rho}_{\mathrm{EC}, T}$ as modelling the energetic curtain of [1] when $T \gg(2 \pi)^{-1}[25]$.

\subsection{Detector}

We consider the response of an inertial detector that crosses the Rindler horizon from $\mathbf{R}$ to $\mathbf{F}$, with the field in the state $\hat{\rho}_{\mathrm{EC}, T}$. The response differs from that in the state $\hat{\rho}_{\mathrm{FW}}$ by the additional term

$$
\Delta_{\mathrm{EC}} \mathcal{F}^{(p)}(\omega)=\int_{-\infty}^{\infty} d \tau^{\prime} \int_{-\infty}^{\infty} d \tau^{\prime \prime} e^{-i \omega\left(\tau^{\prime}-\tau^{\prime \prime}\right)} \chi\left(\tau^{\prime}\right) \chi\left(\tau^{\prime \prime}\right) \partial_{\tau^{\prime}}^{p} \partial_{\tau^{\prime \prime}}^{p} \Delta_{\mathrm{EC}} \mathcal{W}\left(\tau^{\prime}, \tau^{\prime \prime}\right)
$$

where

$$
\Delta_{\mathrm{EC}} \mathcal{W}\left(\tau^{\prime}, \tau^{\prime \prime}\right)= \begin{cases}\frac{1}{4 \pi} \ln \left[\frac{\widetilde{m}\left(\tau^{\prime}-\tau^{\prime \prime}\right)}{\left(\widetilde{m} \tau^{\prime}\right)^{2 \pi T}-\left(\widetilde{m} \tau^{\prime \prime}\right)^{2 \pi T}}\right] & \text { for } \tau^{\prime}>\tau^{\prime \prime}>0 \text { or } \tau^{\prime \prime}>\tau^{\prime}>0 \\ 0 & \text { otherwise }\end{cases}
$$

and $\widetilde{m}:=m_{0} e^{-\beta} . \Delta_{\mathrm{EC}} \mathcal{F}^{(p)}$ is clearly finite for all $T$ and $p$. 
We are interested in the limit of large $T$. Proceeding as in section 5 , and using the techniques of appendix $\mathrm{B}$, we find that the asymptotic large $T$ forms of $\Delta_{\mathrm{EC}} \mathcal{F}^{(0)}$ and $\Delta_{\mathrm{EC}} \mathcal{F}^{(1)}$ are

$$
\begin{aligned}
& \Delta_{\mathrm{EC}} \mathcal{F}^{(0)}(\omega)=-T \int_{0}^{\infty} d s \cos (\omega s) \int_{s}^{\infty} d u \ln (\widetilde{m} u) \chi(u) \chi(u-s)+O\left(T^{0}\right), \\
& \Delta_{\mathrm{EC}} \mathcal{F}^{(1)}(\omega)=T[ {[\chi(0)]^{2} \ln \left(\frac{|\omega| e^{\gamma}}{\widetilde{m}}\right)+\chi(0) \int_{0}^{\infty} d s \cos (\omega s) \frac{\chi(0)-\chi(s)}{s} } \\
&\left.\quad-\int_{0}^{\infty} d u \ln (\widetilde{m} u) \chi^{\prime}(u) \chi(u)\right]+O\left(T^{0}\right) \quad \text { for } \omega \neq 0, \\
& \Delta_{\mathrm{EC}} \mathcal{F}^{(1)}(0)=T \int_{0}^{\infty} d u \ln (\widetilde{m} u) \chi^{\prime}(u)[\chi(0)-\chi(u)]+O\left(T^{0}\right) .
\end{aligned}
$$

The leading behaviour is hence linear in $T$. When $|\omega|$ is large, we may use the techniques of appendix A to show that

$$
\begin{aligned}
\Delta_{\mathrm{EC}} \mathcal{F}^{(0)}(\omega)= & T\left[\frac{[\chi(0)]^{2}}{\omega^{2}} \ln \left(\frac{|\omega| e^{\gamma}}{\widetilde{m}}\right)-\frac{1}{\omega^{2}} \int_{0}^{\infty} d u \ln (\widetilde{m} u) \chi^{\prime}(u) \chi(u)+O\left(\frac{\ln (|\omega|)}{\omega^{4}}\right)\right] \\
& +O\left(T^{0}\right), \\
\Delta_{\mathrm{EC}} \mathcal{F}^{(1)}(\omega)= & T\left[[\chi(0)]^{2} \ln \left(\frac{|\omega| e^{\gamma}}{\widetilde{m}}\right)-\int_{0}^{\infty} d u \ln (\widetilde{m} u) \chi^{\prime}(u) \chi(u)+\chi(0) O\left(\omega^{-2}\right)\right] \\
& +O\left(T^{0}\right) .
\end{aligned}
$$

If $\chi(0)=0$, the leading $\omega$-dependence at large $|\omega|$ drops out from the $T$-term in (6.6a), and the $T$-term in (6.6b) becomes independent of $\omega$.

We conclude that the response can be made arbitrarily large by increasing $T$, and the part of this response that is dominant at large $|\omega|$ comes from the horizon-crossing moment.

\section{Summary and concluding remarks}

We have shown that a two-level UDW detector in $(1+1)$-dimensional Minkowski spacetime, coupled linearly to a massless scalar field or its proper time derivative, has a finite response on crossing inertially the Rindler horizon in a firewall-type quantum state in which the Minkowski vacuum correlations between the right and left Rindler wedges have been fully severed. In the limit of a large detector energy gap $\omega$, the leading contribution to the difference from the Minkowski vacuum response is proportional to $[\chi(0)]^{2} \omega^{-2} \ln (|\omega|)$ for the non-derivative detector and to $[\chi(0)]^{2} \ln (|\omega|)$ for the derivative-coupling detector, where $\chi(0)$ is the coupling strength at the horizon-crossing moment. The same leading contributions arise also in the limit of adiabatic switching. If the detector operates both before and after the horizon-crossing moment but not at the horizon-crossing moment, and the coupling strength changes smoothly in time, the effect is weaker: for a detector whose coupling vanishes in any open interval containing the horizon-crossing moment, the difference from the Minkowski vacuum response dies off at large $|\omega|$ faster than any inverse power of $\omega$. 
Our construction of the Rindler firewall state $\hat{\rho}_{\mathrm{FW}}$ relied on the fact that the rightmoving and left-moving components of a massless field are decoupled in $1+1$ dimensions. (Related consequences of this decoupling for past-future correlations have been investigated in $[31,32].) \quad \hat{\rho}_{\mathrm{FW}}$ is not Hadamard at the Rindler horizon, and we found that the Wightman function of $\hat{\rho}_{\mathrm{FW}}$ contains a heightened version of the $(1+1)$-dimensional infrared ambiguity. In particular we found that the response of the derivative-coupling detector is ambiguous by an additive Lorentz-noninvariant constant, even though this detector is free from infrared ambiguities in Hadamard states [18]. It could be interesting to investigate whether such ambiguities are present for the derivative-coupling detector in firewall-type states in which a severing of correlations evolves from an initially regular state by some dynamical mechanism.

We emphasise that $\hat{\rho}_{\mathrm{FW}}$ is undoubtedly singular at the Rindler horizon, as seen from the non-Hadamard form of the Wightman function, and from the way in which the detector's response hinges on the coupling strength at the horizon-crossing moment. $\hat{\rho}_{\mathrm{FW}}$ is hence qualitatively different from an evaporating $(1+1)$-dimensional black hole in the CGHS model, where the outcome is a long-lived remnant [33], and from a $(1+1)$-dimensional moving-mirror system that models a remnant [34]. We have not attempted to characterise the singularity in $\hat{\rho}_{\mathrm{FW}}$ in terms of a stress-energy tensor, or by other means that might indicate how the spacetime responds to the singularity when allowed to become dynamical. However, our main observation is that when the spacetime is assumed to be unaffected by the singularity in $\hat{\rho}_{\mathrm{FW}}$, the response of the detector that falls across the horizon is, while sudden, nevertheless finite.

Our UDW detector had two internal states. If the detector's internal Hilbert space is generalised to that of a harmonic oscillator, it would be usual to take $\mu$ in (2.1) to be the oscillator's position operator, $\mu(\tau)=e^{i \Omega \tau} d^{\dagger}+e^{-i \Omega \tau} d$, where $\Omega>0$ is the oscillator's angular frequency and $\left(d, d^{\dagger}\right)$ are the annihilation and creation operator pair $[13,14,16]$. For the non-derivative detector in $3+1$ dimensions, this choice for $\mu$ models the $\boldsymbol{p} \cdot \boldsymbol{A}$ term by which an atomic electron couples to the quantised electromagnetic field when there is no angular momentum exchange [23, 24]. With this choice, $\mu$ has nonvanishing matrix elements only between neighbouring energy eigenstates, and the only nonvanishing firstorder transition probabilities from detector state $|n\rangle_{D}$ are to detector states $|n+1\rangle_{D}$ and $|n-1\rangle_{D}$, given by our formulas with $\omega= \pm \Omega$. The conclusion about a finite detector response on crossing the firewall hence still holds. If however $\mu$ were chosen to have matrix elements of equal magnitude between each pair of the harmonic oscillator eigenstates, the sum of the first-order transition probabilities from state $|n\rangle_{D}$ to all other states would diverge for the derivative-coupling detector, because of the leading term proportional to $\ln (|\Delta n|)$ at large $\Delta n$, but be still finite for the non-derivative detector, because the leading term is only proportional to $(\Delta n)^{-2} \ln (|\Delta n|)$.

We considered also a generalisation of $\hat{\rho}_{\mathrm{FW}}$ in which excitations are added behind the Rindler horizon in a way that has been suggested [25] to model the energetic curtain of [1]. We found that the response is qualitatively similar to that in $\hat{\rho}_{\mathrm{FW}}$ but can be made arbitrarily large by increasing the temperature-like parameter that characterises the added excitations. 
Finally, recall that the short-distance behaviour of the Wightman function becomes more singular as the spacetime dimension increases. One may hence expect an UDW detector in dimensions higher than $1+1$ to react to a firewall more violently [22]. However, the short-distance behaviour of the derivative-coupling detector in $1+1$ dimensions is similar to that of the non-derivative detector in $3+1$ dimensions $[18,20,21]$. This suggests that our results for the $1+1$ derivative-coupling UDW detector may faithfully reflect the response of a non-derivative UDW detector that crosses a $(3+1)$-dimensional firewall.

\section{Acknowledgments}

I thank Don Marolf for asking how a detector responds in the state $\hat{\rho}_{\mathrm{FW}}$ and for helpful correspondence, and Sam Braunstein for asking how a detector responds in the state $\hat{\rho}_{\mathrm{EC}, T}$ considered in section 6. I thank Doyeol Ahn and Paul Nation for the invitation to present an early version of this work at the meeting RQI North 2014, Seoul, Korea, 30 June3 July 2014, and several participants, including Eric Brown, Nick Menicucci, Don Page and Bill Unruh, for useful comments. I thank an anonymous referee for helpful suggestions. This work was supported in part by STFC (Theory Consolidated Grant ST/J000388/1).

\section{A Asymptotics at large $|\omega|$}

In this appendix we verify the asymptotic large $|\omega|$ expressions (3.4), (5.5) and (5.6). We assume $\omega \neq 0$, and we denote by $O^{\infty}\left(\omega^{-1}\right)$ a quantity that vanishes faster than any inverse power of $\omega$ as $|\omega| \rightarrow \infty$.

\section{A.1 Minkowski vacuum response}

Consider in $\mathcal{F}_{\left|0_{M}\right\rangle}^{(1)}$ (3.3b). Repeated integration by parts, integrating the trigonometric factor [35], shows that the second term in $(3.3 \mathrm{~b})$ is $O^{\infty}\left(\omega^{-1}\right)$. This gives $(3.4 \mathrm{~b})$ in the main text.

Consider then in $\mathcal{F}_{\left|0_{M}\right\rangle}^{(0)}(3.3 \mathrm{a})$. We write

$$
\begin{aligned}
{ }^{\text {in }} \mathcal{F}_{\left|0_{M}\right\rangle}^{(0)}(\omega) & ={ }^{\text {in }} \mathcal{F}_{1}^{(0)}(\omega)+{ }^{\text {in }} \mathcal{F}_{2}^{(0)}(\omega), \\
{ }^{\text {in }} \mathcal{F}_{1}^{(0)}(\omega) & =-\frac{1}{2} \int_{0}^{\infty} d s \sin (\omega s) H(s), \\
{ }^{\text {in }} \mathcal{F}_{2}^{(0)}(\omega) & =-\frac{1}{\pi} \int_{0}^{\infty} d s \cos (\omega s) \ln \left(m_{0} s\right) H(s),
\end{aligned}
$$

where $H(s):=\int_{-\infty}^{\infty} d u \chi(u) \chi(u-s) . H$ is a smooth function of compact support, it is even, and integration by parts shows that $H^{(2 k)}(0)=(-1)^{k} \int_{-\infty}^{\infty} d u\left[\chi^{(k)}(u)\right]^{2}$ for $k=0,1,2, \ldots$

For in ${ }^{(0)}{ }_{1}^{(0)}$, repeated integration by parts in (A.1b) gives

$$
{ }^{\text {in }} \mathcal{F}_{1}^{(0)}(\omega)=-\frac{1}{2} \sum_{r=0}^{k}(-1)^{r} \frac{H^{(2 r)}(0)}{\omega^{2 r+1}}+O\left(\frac{1}{\omega^{2 k+3}}\right), \quad k=0,1,2, \ldots
$$


For ${ }^{\text {in }} \mathcal{F}_{2}^{(0)}$, integrating (A.1c) by parts twice gives

$$
\begin{aligned}
\text { in } \mathcal{F}_{2}^{(0)}(\omega)= & \frac{1}{\pi \omega} \int_{0}^{\infty} d s \frac{\sin (\omega s)}{s} H(s)+\frac{1}{\pi \omega^{2}} \int_{0}^{\infty} d s \cos (\omega s) \frac{H^{\prime}(s)}{s} \\
& +\frac{1}{\pi \omega^{2}} \int_{0}^{\infty} d s \cos (\omega s) \ln \left(m_{0} s\right) H^{\prime \prime}(s)
\end{aligned}
$$

In the first term in (A.3) we write $H(s)=H(0)+[H(s)-H(0)]$, we use in the part proportional to $H(0)$ the identity $\int_{0}^{\infty} d x x^{-1} \sin x=\pi / 2$, and we estimate the remainder by repeated integration by parts, finding that this term equals $\frac{1}{2} H(0) /|\omega|+O^{\infty}\left(\omega^{-1}\right)$. The second term in (A.3) is $O^{\infty}\left(\omega^{-1}\right)$, again using repeated integration by parts. The last term in (A.3) has the same form as (A.1c) but with $H \rightarrow H^{\prime \prime}$ and an overall factor $-1 / \omega^{2}$. Proceeding recursively, we hence obtain

$$
\text { in } \mathcal{F}_{2}^{(0)}(\omega)=\frac{1}{2} \sum_{r=0}^{k}(-1)^{r} \frac{H^{(2 r)}(0)}{|\omega|^{2 r+1}}+O\left(\frac{1}{|\omega|^{2 k+3}}\right), \quad k=0,1,2, \ldots
$$

Substituting (A.2) and (A.4) into (A.1a), and using the values of $H^{(2 k)}(0)$ found above, gives $(3.4 \mathrm{a})$ in the main text.

\section{A.2 Firewall response}

Consider $\Delta \mathcal{F}^{(1)}$ (5.3b). The large $|\omega|$ expansion of the second term can be obtained by repeated integration by parts, integrating the trigonometric term [35]. When $\chi(0) \neq 0$, the leading terms are shown in (5.5b). When $\chi(0)=0$, it follows from the non-negativity of $\chi$ that $\chi^{\prime}(0)=0$, and the expansion starts as shown in (5.6b).

Consider then $\Delta \mathcal{F}^{(0)}(5.3 \mathrm{a})$. We write

$$
\begin{aligned}
& \Delta \mathcal{F}^{(0)}(\omega)=\Delta \mathcal{F}_{1}^{(0)}(\omega)+\Delta \mathcal{F}_{2}^{(0)}(\omega), \\
& \Delta \mathcal{F}_{1}^{(0)}(\omega)=\frac{1}{4} \int_{0}^{\infty} d s \sin (\omega s) G(s), \\
& \Delta \mathcal{F}_{2}^{(0)}(\omega)=\frac{1}{2 \pi} \int_{0}^{\infty} d s \cos (\omega s) \ln (\widetilde{m} s) G(s),
\end{aligned}
$$

where $\widetilde{m}:=m_{0} e^{\eta \beta}$ and $G(s):=\int_{0}^{s} d u \chi(u) \chi(u-s) . G$ is a smooth function of compact support, it is odd, and we have $G^{\prime}(0)=[\chi(0)]^{2}, G^{(3)}(0)=2 \chi(0) \chi^{\prime \prime}(0)-\left[\chi^{\prime}(0)\right]^{2}$ and $G^{(5)}(0)=2 \chi(0) \chi^{(4)}(0)-2 \chi^{\prime}(0) \chi^{(3)}(0)+\left[\chi^{\prime \prime}(0)\right]^{2}$.

For $\Delta \mathcal{F}_{1}^{(0)}$, repeated integration by parts in (A.5b) gives $\Delta \mathcal{F}_{1}^{(0)}(\omega)=O^{\infty}\left(\omega^{-1}\right)$.

For $\Delta \mathcal{F}_{2}^{(0)}$, integration by parts in (A.5c) gives

$$
\Delta \mathcal{F}_{2}^{(0)}(\omega)=-\frac{1}{2 \pi \omega} \int_{0}^{\infty} d s \sin (\omega s) \frac{G(s)}{s}-\frac{1}{2 \pi \omega} \int_{0}^{\infty} d s \sin (\omega s) \ln (\widetilde{m} s) G^{\prime}(s)
$$


To handle the second term in (A.6), we introduce a cutoff $\epsilon>0$ and observe that

$$
\begin{aligned}
\omega \int_{\epsilon}^{\infty} d s \sin (\omega s) \ln (\widetilde{m} s) G^{\prime}(s)= & \cos (\omega \epsilon) \ln (\widetilde{m} \epsilon) G^{\prime}(\epsilon)-G^{\prime}(0) \operatorname{Ci}(\epsilon|\omega|) \\
& +\int_{\epsilon}^{\infty} d s \cos (\omega s) \frac{G^{\prime}(s)-G^{\prime}(0)}{s} \\
& +\int_{\epsilon}^{\infty} d s \cos (\omega s) \ln (\widetilde{m} s) G^{\prime \prime}(s)
\end{aligned}
$$

first integrating by parts and then subtracting and adding $G^{\prime}(0) \mathrm{Ci}(|\omega| \epsilon)$, where $\mathrm{Ci}$ is the cosine integral function in the notation of [36]. The limit $\epsilon \rightarrow 0_{+}$in (A.7) can be taken using the small argument form of $\mathrm{Ci}$ [36], and substituting the result in (A.6) yields

$$
\begin{aligned}
\Delta \mathcal{F}_{2}^{(0)}(\omega)= & -\frac{1}{2 \pi \omega} \int_{0}^{\infty} d s \sin (\omega s) \frac{G(s)}{s}+\frac{G^{\prime}(0)}{2 \pi \omega^{2}} \ln \left(|\omega| e^{\gamma} / \widetilde{m}\right) \\
& -\frac{1}{2 \pi \omega^{2}} \int_{0}^{\infty} d s \cos (\omega s) \frac{G^{\prime}(s)-G^{\prime}(0)}{s} \\
& -\frac{1}{2 \pi \omega^{2}} \int_{0}^{\infty} d s \cos (\omega s) \ln (\widetilde{m} s) G^{\prime \prime}(s),
\end{aligned}
$$

where $\gamma$ is Euler's constant. Repeated integration by parts gives for the first term in (A.8) an expansion in inverse powers of $\omega^{2}$, and the same technique shows that the third term in (A.8) is $O^{\infty}\left(\omega^{-1}\right)$. We find

$$
\begin{aligned}
\Delta \mathcal{F}_{2}^{(0)}(\omega)= & -\frac{1}{2 \pi \omega^{2}} \int_{0}^{\infty} d s \cos (\omega s) \ln (\widetilde{m} s) G^{\prime \prime}(s)+\frac{G^{\prime}(0)}{2 \pi \omega^{2}} \ln \left(|\omega| e^{\gamma-1} / \widetilde{m}\right) \\
& +\frac{1}{2 \pi} \sum_{r=2}^{k}(-1)^{r} \frac{G^{(2 r-1)}(0)}{(2 r-1) \omega^{2 r}}+O\left(\frac{1}{\omega^{2 k+2}}\right), \quad k=2,3,4, \ldots
\end{aligned}
$$

Now, the first term in (A.9) has the same form as (A.5c) but with $G \rightarrow G^{\prime \prime}$ and an overall factor $-1 / \omega^{2}$, and we may proceed with $\Delta \mathcal{F}_{2}^{(0)}$ recursively. Collecting, we find for $\Delta \mathcal{F}^{(0)}$ the asymptotic large $|\omega|$ expansion

$$
\begin{aligned}
\Delta \mathcal{F}^{(0)}(\omega) \sim & \frac{1}{2 \pi} \ln \left(|\omega| e^{\gamma-1} / \widetilde{m}\right)\left(\frac{G^{\prime}(0)}{\omega^{2}}-\frac{G^{(3)}(0)}{\omega^{4}}+\frac{G^{(5)}(0)}{\omega^{6}}-\frac{G^{(7)}(0)}{\omega^{8}}+\cdots\right) \\
& +\frac{1}{2 \pi}\left(\frac{\frac{1}{3} G^{(3)}(0)}{\omega^{4}}-\frac{\left(\frac{1}{3}+\frac{1}{5}\right) G^{(5)}(0)}{\omega^{6}}+\frac{\left(\frac{1}{3}+\frac{1}{5}+\frac{1}{7}\right) G^{(7)}(0)}{\omega^{8}}-\cdots\right) .
\end{aligned}
$$

Equations (5.5a) and (5.6a) in the main text follow from (A.10) by inserting the values of $G^{\prime}(0), G^{(3)}(0)$ and $G^{(5)}(0)$ found above.

\section{B Evaluation of $\Delta \mathcal{F}^{(0)}$ and $\Delta \mathcal{F}^{(1)}$}

In this appendix we verify formulas (5.3) for $\Delta \mathcal{F}^{(0)}$ and $\Delta \mathcal{F}^{(1)}$. We write $\widetilde{m}:=m_{0} e^{\eta \beta}$, $Q(\tau):=e^{-i \omega \tau} \chi(\tau)$ and $Q^{\prime}(\tau):=\frac{d}{d \tau} Q(\tau)$. 


\section{B.1 $\Delta \mathcal{F}^{(0)}$}

Starting from (5.1) with $p=0$, we have

$$
\begin{aligned}
\Delta \mathcal{F}^{(0)}(\omega) & =\int_{-\infty}^{\infty} d \tau^{\prime} \int_{-\infty}^{\infty} d \tau^{\prime \prime} Q\left(\tau^{\prime}\right) \overline{Q\left(\tau^{\prime \prime}\right)} \Delta \mathcal{W}\left(\tau^{\prime}, \tau^{\prime \prime}\right) \\
& =\operatorname{Re} \int_{0}^{\infty} d \tau^{\prime} \int_{-\infty}^{0} d \tau^{\prime \prime}\left\{(2 \pi)^{-1} \ln \left[\widetilde{m}\left(\tau^{\prime}-\tau^{\prime \prime}\right)\right]+\frac{1}{4} i\right\} Q\left(\tau^{\prime}\right) \overline{Q\left(\tau^{\prime \prime}\right)}
\end{aligned}
$$

using (5.2) for $\Delta \mathcal{W}$ and interchanging the names of $\tau^{\prime}$ and $\tau^{\prime \prime}$ in the region where originally $\tau^{\prime}<0<\tau^{\prime \prime}$. Writing $u:=\tau^{\prime}$ and $\tau^{\prime \prime}=u-s$, intechanging the integration order, and using $Q(\tau)=e^{-i \omega \tau} \chi(\tau)$, we obtain

$$
\Delta \mathcal{F}^{(0)}(\omega)=\int_{0}^{\infty} d s\left[\frac{1}{4} \sin (\omega s)+(2 \pi)^{-1} \cos (\omega s) \ln (\widetilde{m} s)\right] \int_{0}^{s} d u \chi(u) \chi(u-s),
$$

which is equation (5.3a) in the main text.

\section{B.2 $\Delta \mathcal{F}^{(1)}$}

Starting from (5.1) with $p=1$, we have

$$
\begin{aligned}
\Delta \mathcal{F}^{(1)}(\omega) & =\int_{-\infty}^{\infty} d \tau^{\prime} \int_{-\infty}^{\infty} d \tau^{\prime \prime} Q^{\prime}\left(\tau^{\prime}\right) \overline{Q^{\prime}\left(\tau^{\prime \prime}\right)} \Delta \mathcal{W}\left(\tau^{\prime}, \tau^{\prime \prime}\right) \\
& =\frac{1}{2 \pi} \operatorname{Re} \int_{0}^{\infty} d \tau^{\prime} \int_{-\infty}^{0} d \tau^{\prime \prime} \ln \left[\widetilde{m}\left(\tau^{\prime}-\tau^{\prime \prime}\right)\right] Q^{\prime}\left(\tau^{\prime}\right) \overline{Q^{\prime}\left(\tau^{\prime \prime}\right)}
\end{aligned}
$$

first integrating the distributional derivatives by parts, then using (5.2) for $\Delta \mathcal{W}$ and noting that the contributions from the $\pm \frac{1}{8} i$ terms in (5.2) cancel, and finally interchanging the names of $\tau^{\prime}$ and $\tau^{\prime \prime}$ in the region where originally $\tau^{\prime}<0<\tau^{\prime \prime}$. Writing $u:=\tau^{\prime}$ and $\tau^{\prime \prime}=u-s$, and intechanging the integration order, we obtain

$$
\Delta \mathcal{F}^{(1)}(\omega)=\frac{1}{2 \pi} \operatorname{Re} \int_{0}^{\infty} d s \ln (\widetilde{m} s) \int_{0}^{s} d u Q^{\prime}(u) \overline{Q^{\prime}(u-s)} .
$$

Using in (B.4) the identity

$$
\int_{0}^{s} d u Q^{\prime}(u) \overline{Q^{\prime}(u-s)}=\frac{d}{d s}\left(Q(0) \overline{Q(-s)}+\int_{0}^{s} d u Q(u) \overline{Q^{\prime}(u-s)}\right),
$$

separating the two terms and integrating the second term by parts, we find

$$
\begin{aligned}
& \Delta \mathcal{F}^{(1)}(\omega)=\Delta \mathcal{F}_{1}^{(1)}(\omega)+\Delta \mathcal{F}_{2}^{(1)}(\omega) \\
& \Delta \mathcal{F}_{1}^{(1)}(\omega)=\frac{\chi(0)}{2 \pi} \int_{0}^{\infty} d s \ln (\widetilde{m} s) \frac{d}{d s}[\cos (\omega s) \chi(-s)], \\
& \Delta \mathcal{F}_{2}^{(1)}(\omega)=-\frac{1}{2 \pi} \operatorname{Re} \int_{0}^{\infty} \frac{d s}{s} \int_{0}^{s} d u Q(u) \overline{Q^{\prime}(u-s)} .
\end{aligned}
$$

Consider first $\Delta \mathcal{F}_{1}^{(1)}$ (B.6b). When $\omega=0$, (B.6b) reduces to

$$
\Delta \mathcal{F}_{1}^{(1)}(0)=-\frac{\chi(0)}{2 \pi} \int_{0}^{\infty} d s \ln (\widetilde{m} s) \chi^{\prime}(-s) .
$$


When $\omega \neq 0$, we introduce a cutoff $\epsilon>0$ and write

$$
\begin{aligned}
\int_{\epsilon}^{\infty} d s \ln (\widetilde{m} s) \frac{d}{d s}[\cos (\omega s) \chi(-s)]= & -\ln (\widetilde{m} \epsilon) \cos (\omega \epsilon) \chi(-\epsilon)-\int_{\epsilon}^{\infty} \frac{d s}{s} \cos (\omega s) \chi(-s) \\
= & -\ln (\widetilde{m} \epsilon) \cos (\omega \epsilon) \chi(-\epsilon)+\chi(0) \operatorname{Ci}(|\omega| \epsilon) \\
& +\int_{\epsilon}^{\infty} d s \cos (\omega s) \frac{[\chi(0)-\chi(-s)]}{s}
\end{aligned}
$$

integrating by parts and adding and subtracting $\chi(0) \mathrm{Ci}(|\omega| \epsilon)$. Using the small argument form of $\mathrm{Ci}$ to take the limit [36], we find

$$
\Delta \mathcal{F}_{1}^{(1)}(\omega)=\frac{[\chi(0)]^{2}}{2 \pi} \ln \left(|\omega| e^{\gamma} / \widetilde{m}\right)+\frac{\chi(0)}{2 \pi} \int_{0}^{\infty} d s \cos (\omega s) \frac{[\chi(0)-\chi(-s)]}{s},
$$

where $\gamma$ is Euler's constant.

Consider then $\Delta \mathcal{F}_{2}^{(1)}$ (B.6c). Using in (B.6c) the identity

$$
-\operatorname{Re} \int_{0}^{s} d u Q(u) \overline{Q^{\prime}(u-s)}=-\cos (\omega s) \chi(0) \chi(s)+\frac{d}{d s} \int_{0}^{s} d u \cos (\omega s) \chi(u) \chi(u-s)
$$

and integrating the second term in (B.10) by parts, we find

$$
\begin{aligned}
\Delta \mathcal{F}_{2}^{(1)}(\omega)=\frac{1}{2 \pi} \lim _{\epsilon \rightarrow 0_{+}}\{ & -\frac{1}{\epsilon} \int_{0}^{\epsilon} d u \chi(u) \chi(u-\epsilon) \\
& \left.+\int_{\epsilon}^{\infty} d s \cos (\omega s)\left[-\frac{\chi(0) \chi(s)}{s}+\frac{1}{s^{2}} \int_{0}^{s} d u \chi(u) \chi(u-s)\right]\right\} \\
=-\frac{[\chi(0)]^{2}}{2 \pi} & +\frac{1}{2 \pi} \int_{0}^{\infty} d s \cos (\omega s)\left[-\frac{\chi(0) \chi(s)}{s}+\frac{1}{s^{2}} \int_{0}^{s} d u \chi(u) \chi(u-s)\right] .
\end{aligned}
$$

For $\omega \neq 0$, combining (B.9) and (B.11) gives (5.3b) in the main text.

For $\omega=0$, we set $\omega=0$ in (B.11), we add and subtract under the $s$-integral the term $\chi(0)[\chi(-s)-\chi(s)](2 s)^{-1}$, and we integrate the added term by parts. Combining with (B.7) gives $(5.3 \mathrm{c})$ in the main text.

Open Access. This article is distributed under the terms of the Creative Commons Attribution License (CC-BY 4.0), which permits any use, distribution and reproduction in any medium, provided the original author(s) and source are credited.

\section{References}

[1] S.L. Braunstein, S. Pirandola and K. Życzkowski, Better late than never: information retrieval from black holes, Phys. Rev. Lett. 110 (2013) 101301 [arXiv:0907.1190] [INSPIRE].

[2] S.D. Mathur, The information paradox: a pedagogical introduction, Class. Quant. Grav. 26 (2009) 224001 [arXiv:0909.1038] [INSPIRE].

[3] A. Almheiri, D. Marolf, J. Polchinski and J. Sully, Black holes: complementarity or firewalls?, JHEP 02 (2013) 062 [arXiv:1207.3123] [INSPIRE]. 
[4] S.W. Hawking, Particle creation by black holes, Commun. Math. Phys. 43 (1975) 199 [Erratum ibid. 46 (1976) 206] [INSPIRE].

[5] L. Susskind, Black hole complementarity and the Harlow-Hayden conjecture, arXiv:1301.4505 [INSPIRE].

[6] A. Almheiri, D. Marolf, J. Polchinski, D. Stanford and J. Sully, An apologia for firewalls, JHEP 09 (2013) 018 [arXiv: 1304.6483] [INSPIRE].

[7] D.N. Page, Excluding black hole firewalls with extreme cosmic censorship, JCAP 06 (2014) 051 [arXiv: 1306.0562] [INSPIRE].

[8] W.G. Unruh, Notes on black hole evaporation, Phys. Rev. D 14 (1976) 870 [InSPIRE].

[9] L.C.B. Crispino, A. Higuchi and G.E.A. Matsas, The Unruh effect and its applications, Rev. Mod. Phys. 80 (2008) 787 [arXiv:0710.5373] [InSPIRE].

[10] B.S. DeWitt, Quantum gravity: the new synthesis, in General relativity: an Einstein centenary survey, S.W. Hawking and W. Israel eds., Cambridge University Press, Cambridge U.K. (1979), pg. 680.

[11] N.D. Birrell and P.C.W. Davies, Quantum fields in curved space, Cambridge University Press, Cambridge U.K. (1982) [INSPIRE].

[12] R.M. Wald, Quantum field theory in curved spacetime and black hole thermodynamics, University of Chicago Press, Chicago U.S.A. (1994).

[13] D.J. Raine, D.W. Sciama and P.G. Grove, Does a uniformly accelerated quantum oscillator radiate?, Proc. Roy. Soc. Lond. A 435 (1991) 205.

[14] A. Raval, B.L. Hu and J. Anglin, Stochastic theory of accelerated detectors in a quantum field, Phys. Rev. D 53 (1996) 7003 [gr-qc/9510002] [InSPIRE].

[15] P.C.W. Davies and A.C. Ottewill, Detection of negative energy: 4-dimensional examples, Phys. Rev. D 65 (2002) 104014 [gr-qc/0203003] [INSPIRE].

[16] Q. Wang and W.G. Unruh, Motion of a mirror under infinitely fluctuating quantum vacuum stress, Phys. Rev. D 89 (2014) 085009 [arXiv:1312.4591] [INSPIRE].

[17] E. Martín-Martínez and J. Louko, Particle detectors and the zero mode of a quantum field, Phys. Rev. D 90 (2014) 024015 [arXiv: 1404.5621] [INSPIRE].

[18] B.A. Juárez-Aubry and J. Louko, Onset and decay of the 1+1 Hawking-Unruh effect: what the derivative-coupling detector saw, Class. Quant. Grav., in press [arXiv:1406.2574] [INSPIRE].

[19] Y. Décanini and A. Folacci, Hadamard renormalization of the stress-energy tensor for a quantized scalar field in a general spacetime of arbitrary dimension, Phys. Rev. D 78 (2008) 044025 [gr-qc/0512118] [INSPIRE].

[20] A. Satz, Then again, how often does the Unruh-DeWitt detector click if we switch it carefully?, Class. Quant. Grav. 24 (2007) 1719 [gr-qc/0611067] [INSPIRE].

[21] J. Louko and A. Satz, Transition rate of the Unruh-DeWitt detector in curved spacetime, Class. Quant. Grav. 25 (2008) 055012 [arXiv:0710.5671] [INSPIRE].

[22] L. Hodgkinson and J. Louko, How often does the Unruh-DeWitt detector click beyond four dimensions?, J. Math. Phys. 53 (2012) 082301 [arXiv:1109.4377] [INSPIRE]. 
[23] E. Martín-Martínez, M. Montero and M. del Rey, Wavepacket detection with the Unruh-DeWitt model, Phys. Rev. D 87 (2013) 064038 [arXiv:1207.3248] [InSPIRE].

[24] Á.M. Alhambra, A. Kempf and E. Martín-Martínez, Casimir forces on atoms in optical cavities, Phys. Rev. A 89 (2014) 033835 [arXiv:1311.7619].

[25] S.L. Braunstein, private communication (2014).

[26] C.J. Fewster, A general worldline quantum inequality, Class. Quant. Grav. 17 (2000) 1897 [gr-qc/9910060] [INSPIRE].

[27] W. Junker and E. Schrohe, Adiabatic vacuum states on general space-time manifolds: definition, construction and physical properties, Ann. Poincaré Phys. Theor. 3 (2002) 1113 [math-ph/0109010] [INSPIRE].

[28] L. Hörmander, The analysis of linear partial differential operators I (distribution theory and Fourier analysis), 2nd edition, Springer, Berlin Germany (1990).

[29] L. Hörmander, Fourier integral operators. I, Acta Mathematica 127 (1971) 79, reprinted in J. Brüning and V.W. Guillemin eds., Mathematics past and present. Fourier integral operators, Springer, Berlin Germany (1994).

[30] B. Reznik, A. Retzker and J. Silman, Violating Bell's inequalities in vacuum, Phys. Rev. A 71 (2005) 042104 [quant-ph/0310058].

[31] S.J. Olson and T.C. Ralph, Entanglement between the future and past in the quantum vacuum, Phys. Rev. Lett. 106 (2011) 110404 [arXiv:1003.0720] [INSPIRE].

[32] S.J. Olson and T.C. Ralph, Extraction of timelike entanglement from the quantum vacuum, Phys. Rev. A 85 (2012) 012306 [arXiv:1101.2565] [InSPIRE].

[33] A. Almheiri and J. Sully, An uneventful horizon in two dimensions, JHEP 02 (2014) 108 [arXiv: 1307.8149] [INSPIRE].

[34] M. Hotta, J. Matsumoto and K. Funo, Black hole firewalls require huge energy of measurement, Phys. Rev. D 89 (2014) 124023 [arXiv:1306.5057] [INSPIRE].

[35] R. Wong, Asymptotic approximations of integrals, Society for Industrial and Applied Mathematics, Philadelphia U.S.A. (2001).

[36] NIST Digital Library of Mathematical Functions, http://dlmf.nist.gov/, release 1.0.6 (2013). 